\title{
Substâncias tóxicas e tentativas e suicídios: considerações sobre acesso e medidas restritivas
}

\author{
Poisoning and suicide attempts and suicides: considerations \\ on access and restrictive measures
}

\author{
Simone Agadir Santos ${ }^{1}$, Letícia Fortes Legay², Giovanni Marcos Lovisi ${ }^{3}$
}

\begin{abstract}
Resumo
Objetivo: O presente estudo se propôs estimar a frequência do uso das substâncias tóxicas como meio para tentativas e suicídio, como subsídios para a discussão sobre medidas preventivas e de restrição. Métodos: Foram analisados dados presentes nos Sistemas de Informação sobre Mortalidade (SIM) e de Informações Hospitalares do Sistema Único de Saúde (SIH-SUS), período 1998-2009. As variáveis analisadas foram sexo, idade e causa básica (CID-10 X60-X69). Resultados: Cerca de 70,0\% dos indivíduos que tentaram suicídio (SIH-SUS) ingeriram substâncias tóxicas: medicamentos (46,2\%), álcool (29,8\%) e pesticidas (15,1\%). O sexo masculino e o grupo etário de 30-49 anos predominaram. Quanto ao suicídio (SIM), destacaram-se as substâncias medicamentos $(21,7 \%)$ e pesticidas (28,3\%). As mulheres adolescentes predominaram (3:1). A frequência na ingestão de pesticidas não foi muito diferente entre homens $(49,2 \%)$ e mulheres $(42,7 \%)$, quanto ao uso de medicamentos, as mulheres se sobressaíram (28,9 versus 17,3\%). Conclusões: As frequências observadas a partir dos dados oficiais indicaram um perfil jovem nas tentativas e suicídios por intoxicação principalmente por medicamentos psicotrópicos e agrotóxicos. As informações sobre estes meios utilizados permitiram a discussão sobre aceitabilidade social e acessibilidade como determinantes na escolha destes. Constatou-se a inexistência de planos estratégicos específicos para prevenção do suicídio e sim ações pontuais sobre fatores associados.
\end{abstract}

Palavras-chave: tentativa de suicídio; suicídio; intoxicação exógena; sistemas de informação em saúde; vigilância epidemiológica.

\begin{abstract}
Objective: This study aimed to estimate the frequency of the use of toxic substances as methods of suicide attempts and suicide for discussion of preventive measures and restrictions. Methods: Data on the mortality rates due suicide attempts and suicide were collected from the Mortality Information System (SIM) and Hospital Information System in Health System of Brazil (SIH-SUS) between 1998 and 2009. The variables analyzed were age, sex and underlying causes (ICD-10: X60-X69). Results: Around $70.0 \%$ of the attempted suicide cases (SIH-SUS) had ingested toxic substances: medicines (46.2\%), alcohol (29.8\%) and pesticides (15.1\%). The following social-demographic characteristics were predominant in all analyzed cases: men and aged between 30 and 49 years. Regarding the suicide cases (SIM), the most frequently ingest substances were medicines $(21.7 \%)$ and pesticides $(28.3 \%)$, predominating adolescent women (3:1). The frequency of ingestion of pesticides was slightly more frequent in men $(49.2 \%)$ than women $(42.7 \%)$ but women ingested much more medicines than men (28.9 versus $17.3 \%)$. Conclusion: The data indicated a young profile, and allowed the discussion of social acceptability and accessibility as determinants in the choice of methods used in the suicide attempts and suicide by poisoning. The study showed no strategic plans for suicide prevention were present but only specific actions for associated factors.
\end{abstract}

Keywords: suicide, attempted; suicide; poisoning; information systems in health; epidemiologic surveillance.

Trabalho realizado no Instituto de Estudos em Saúde Coletiva da Universidade Federal do Rio de Janeiro (UFRJ) - Rio de Janeiro (RJ), Brasil. 'Doutora em Saúde Coletiva pelo Instituto de Estudos em Saúde Coletiva (IESC) da UFRJ - Rio de Janeiro (RJ), Brasil.

2Doutora em Saúde Pública Pela Universidade de São Paulo (USP) - São Paulo (SP), Brasil.; Professora Associada do IESC/UFRJ - Rio de Janeiro (RJ), Brasil. ${ }^{3}$ Doutor em Saúde Pública pela Escola Nacional de Saúde Pública da Fundação Oswaldo Cruz (FIOCRUZ-ENSP); Professor Associado do IESC/UFRJ - Rio de Janeiro (RJ), Brasil.

Endereço para correspondência: Simone Agadir Santos - Avenida Horacio Macedo, s/n - Ilha do Fundão - Cidade Universitária - CEP: 21941-598 -

Rio de Janeiro (RJ), Brasil - E-mail: simoneagadir@gmail.com

Fonte de financiamento: nenhuma.

Conflito de interesse: nada a declarar. 


\section{INTRODUÇÃO}

A intoxicação exógena se destaca como meio utilizado para a tentativa/suicídio. Dentre as principais substâncias usadas estão os agrotóxicos, com frequências de até $90 \%$ nos países em desenvolvimento, enquanto que o uso de medicamentos chega a $60 \%$ e é mais frequente em países desenvolvidos. No Brasil, a intoxicação exógena é responsável por aproximadamente $70 \%$ dos casos notificados ${ }^{1-9}$.

A prevenção e o controle mais eficaz dessas violências depende da correta classificação. Contudo, a dificuldade dos serviços para identificar adequadamente a intoxicação como intencional e não intencional, assim como a conhecida subnotificação da tentativa/suicídio por motivos socioculturais e econômicos ${ }^{10,11}$ torna difícil o conhecimento real da magnitude dos mesmos. Outro aspecto que dificulta a classificação adequada são os óbitos por causa indeterminada. Neste ponto, é interessante incluir aqui o estudo de Pritchard e Hean ${ }^{12}$, no qual a frequência de óbitos por causa indeterminada em relação aos suicídios em países latino-americanos é maior do que nos países desenvolvidos. Uma possível explicação seriam as atitudes tradicionais religiosas e culturais que favoreceriam a classificação dos óbitos como indeterminados, à semelhança do que ocorre em países islâmicos.

A escolha pelo meio utilizado na tentativa/suicídio abrange aspectos psicossociais, de gênero, aceitabilidade sociocultural, além da disponibilidade no acesso ${ }^{13-15}$. Existem evidências de que medidas restritivas de acesso ao meio têm conduzido à redução da frequência de determinados tipos de suicídio ${ }^{16-18}$.

Raros estudos nacionais ampliaram a discussão sobre medidas restritivas e acesso aos meios utilizados na tentativa/suicídio. O presente estudo teve como objetivo estimar as frequências relativas dos casos de tentativa/suicídio por intoxicação exógena, a partir dos sistemas de informação oficiais e disponíveis para subsidiar a discussão sobre medidas preventivas e de restrição aos meios no cenário atual do comportamento suicida no Brasil.

\section{METODOLOGIA}

Trata-se de um estudo descritivo exploratório sobre as frequências de tentativa e suicídio por intoxicação exógena encontradas nos Sistemas de Informações Hospitalares do Sistema Único de Saúde (SIH-SUS) e sobre Mortalidade (SIM), no período de 1998 a 2009, do Departamento de Informática do Sistema Único de Saúde (DATASUS) do Ministério da Saúde (MS).

Os dois sistemas de informações possuem suas bases de dados disponibilizadas via internet (http://www.datasus.gov.br/), pelo DATASUS, as quais permitem a tabulação online das variáveis de interesse. O recorte feito em ambos os sistemas foi dirigido somente aos casos de intoxicação exógena. Por intoxicação exógena entende-se como consequência da interação entre o agente tóxico e o organismo que ocorre quando o agente tóxico rompe o equilíbrio orgânico, produzindo alterações fisiológicas e bioquímicas. A toxicidade, então, seria o potencial, em maior ou menor grau, do surgimento de um estado patológico como resposta ${ }^{19}$.

Neste artigo, foram consideradas as categorias de causas codificadas como X60-X69 de acordo com a 10ª Classificação Internacional de Doenças (CID-10) ${ }^{20}$ que tratam das causas referentes às intoxicações exógenas, segundo as substâncias tóxicas, para ambos os sistemas. Essas categorias foram agrupadas em: medicamentos (X60-X64); álcool (X65); outros sólidos, líquidos, gases e vapores (X66-X67), pesticidas (X68) e substâncias nocivas não especificadas (X69).

O período 1998-2009 foi escolhido de acordo com a disponibilidade dos dados e melhor qualidade das informações. Importante ressaltar que não foi objetivo do estudo realizar avaliações e análise de cobertura dos sistemas, embora comparações entre os dados a partir das frequências obtidas tenham sido feitas. As variáveis estudadas foram as mesmas nos dois sistemas: sexo e faixas etárias ( 10 a 14, 15 a 19, 20 a 29, 30 a 39, 40 a 49,50 a 59, 60 e mais).

Quanto à concepção dos sistemas, o SIH-SUS fornece dados sobre internações hospitalares provenientes da Autorização de Internação Hospitalar (AIH) emitida por estabelecimento hospitalar público ou conveniado ao SUS ${ }^{21}$. Além das variáveis comuns já citadas, também foram analisados os óbitos hospitalares decorrentes das tentativas. O SIM fornece dados a partir das declarações de óbito coletadas pelas Secretarias Estaduais de Saúde ${ }^{22}$. Ao serem utilizados os dois sistemas, ficaram incluídos todos os óbitos por suicídio (X60-X69).

O programa TabWin do DATASUS/MS foi utilizado para a tabulação dos dados e o cálculo das frequências. Os gráficos e tabelas foram construídos através do aplicativo Excel versão 2007.

\section{Considerações éticas}

Os dados utilizados são de acesso livre via internet (http:// www.datasus.gov.br/).

\section{RESULTADOS}

\section{Perfil das tentativas de suicídio por intoxicação exógena}

No Brasil, foram registradas 112.295 internações devido à tentativa de suicídio, durante o período de 1998 a 2009, sendo que $70,7 \%$ destes indivíduos haviam ingerido substâncias tóxicas, segundo dados do SIH-SUS. 
Observando as categorias de substâncias causadoras das intoxicações, o uso de medicamentos estava presente em 46,2\% das internações, seguido pelo uso do álcool $(29,8 \%)$ e pesticidas (15,1\%). O Gráfico 1 mostra o comportamento das frequências de acordo com as categorias das substâncias utilizadas. Ao longo do período os medicamentos predominaram, em frequência elevada e crescente até 2008. Detalhe interessante é que, tanto em 1998 quanto em 2008, os medicamentos apresentaram seus maiores patamares. Por outro lado o álcool também se destacou como substância utilizada nas tentativas e, embora em frequência menor, foi também constante, com pequena queda em 2005, ascendendo novamente no final do período. $\mathrm{O}$ uso do álcool foi o que apresentou maior aumento $(29,6 \%)$, enquanto que o uso de pesticidas e medicamentos foi reduzido em 27,6 e 7,4\%, respectivamente, ao longo de todo o período. Por outro lado, a categoria substâncias nocivas não especificadas (X69) aumentou em 20,8\%, sendo portanto um indicador ruim.

Como pode ser observado na Tabela 1, 56,4\% das internações são de homens. Agrupando-se os casos por faixas etárias mais frequentes, observou-se que $82,1 \%$ destas internações estão na faixa etária dos adultos em idade produtiva, de 20 a 59 anos. Entre as mulheres, 76,5\% estão na mesma faixa. Entre os adolescentes, $10,0 \%$ são do sexo masculino e $24,4 \%$, do feminino. Quanto às tentativas com uso de medicamentos, as mulheres se destacaram, correspondendo a $63,6 \%$ do total. Por outro lado, a ingestão de álcool para a tentativa foi mais frequente entre os homens $(45,5 \%)$. Novamente, as substâncias responsáveis pelo maior número de óbitos entre as mulheres foram o uso de medicamentos $(46,1 \%)$ e entre os homens, o álcool $(45,5 \%)$. Não se pode deixar também de ressaltar que cerca de 30\% dos óbitos em ambos os sexos, foi devido ao uso de pesticidas.

\section{Perfil dos suicídios por intoxicação exógena}

Enquanto na primeira parte deste estudo foram analisadas as tentativas de suicídio e seus meios a partir dos dados do SIH-SUS, aqui foram avaliadas as mesmas características dos óbitos por suicídio, partindo dos dados do SIM. No período de 1998 a 2009, foram registrados 96.011 óbitos por suicídio, no Brasil. O principal meio utilizado para o suicídio foi enforcamento $(55,1 \%)$, em segundo lugar vem arma de fogo e intoxicação exógena com percentuais semelhantes (15,0\%, em ambos), segundo dados do SIM.

Os óbitos causados por intoxicação exógena foram objeto deste estudo e o Gráfico 2 mostra que a frequência do uso de pesticidas apresentou ligeiras oscilações em 2001 e 2005, mantendo-se praticamente estável durante o período analisado (entre 40 e 50\%). Enquanto isto, a frequência no uso de medicamentos variou entre 19,0 a 25,6\%, sugerindo que, após 2009, o seu uso continuou a se elevar. Devido a sua importância como indicador de qualidade de informação, a categoria substâncias nocivas "não especificadas" (X69) apresentou valores relevantes (acima de 30\%).

Cita-se também a sobremortalidade masculina na faixa etária de 20 a 59 anos (82,0\%). Entre os adolescentes, as mulheres predominaram (3:1), por outro lado, entre os indivíduos de 60 anos e mais, a razão foi de $2: 1$ para os homens. A frequência na ingestão de pesticidas não foi muito diferente entre homens $(49,2 \%)$ e mulheres $(42,7 \%)$; quanto ao uso de medicamentos, as mulheres se sobressaíram $(28,9$ versus $17,3 \%)$ (Tabela 2$)$.
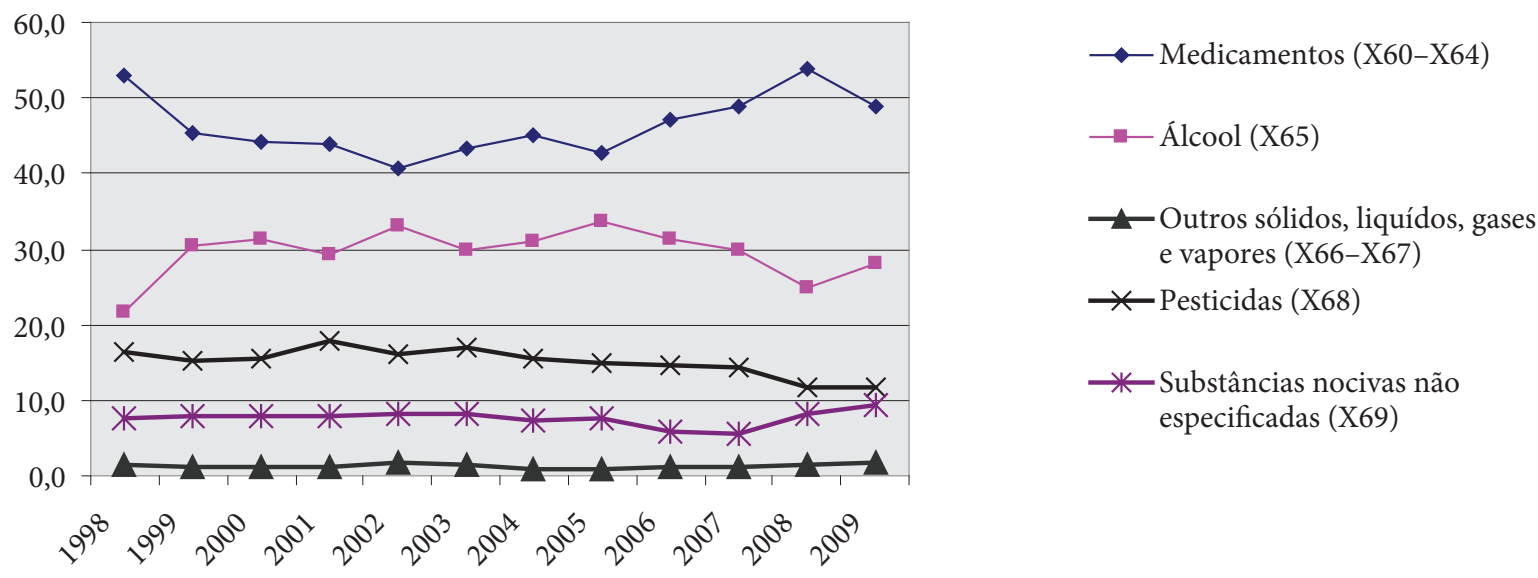

Fonte: SIH-SUS/DATASUS/MS

Gráfico 1. Distribuição da frequência de internação por tentativas de suicídio devido à intoxicação exógena, segundo categorias de substâncias, Brasil, período de 1998 a 2009 
Tabela 1. Distribuição da frequência de internações por tentativas de suicídio devido à intoxicação exógena, segundo sexo, Brasil, período de 1998 a 2009

\begin{tabular}{|c|c|c|}
\hline $\begin{array}{c}\text { Homem } \\
(\mathrm{n}=44.828)\end{array}$ & $\begin{array}{c}\text { Mulher } \\
(\mathrm{n}=34.589)\end{array}$ & $\begin{array}{c}\text { Total } \\
(\mathrm{n}=79.418)\end{array}$ \\
\hline $\mathbf{n}$ & $\%$ & $\mathbf{n}$ \\
\hline
\end{tabular}

Faixa etária (anos)

\begin{tabular}{|c|c|c|c|c|c|c|}
\hline 10 a 14 & 1.371 & 3,1 & 2.183 & 6,3 & 3.554 & 4,5 \\
\hline 15 a $19^{*}$ & 3.114 & 6,9 & 6.114 & 17,7 & 9.229 & 11,6 \\
\hline 20 a 29 & 11.614 & 25,9 & 10.498 & 30,3 & 22.112 & 27,8 \\
\hline 30 a 39 & 13.496 & 30,1 & 8.482 & 24,5 & 21.978 & 27,7 \\
\hline 40 a 49 & 11.418 & 25,5 & 6.021 & 17,4 & 17.439 & 21,9 \\
\hline 50 a 59 & 6.125 & 13,7 & 2.908 & 8,4 & 9.033 & 11,4 \\
\hline 60 e mais & 3.528 & 7,9 & 2.370 & 6,9 & 5.898 & 7,4 \\
\hline \multicolumn{7}{|l|}{ Categorias de causas } \\
\hline Medicamentos $(\mathrm{X} 60-\mathrm{X} 64)^{\star}$ & 14.666 & 32,7 & 22.006 & 63,6 & 36.673 & 46,2 \\
\hline Álcool (X65) & 20.384 & 45,5 & 3.278 & 9,5 & 23.662 & 29,8 \\
\hline $\begin{array}{l}\text { Outros sólidos, liquídos, gases e vapores } \\
\text { (X66-X67) }\end{array}$ & 613 & 1,4 & 439 & 1,3 & 1.052 & 1,3 \\
\hline Pesticidas (X68) & 6.230 & 13,9 & 5.730 & 16,6 & 11.960 & 15,1 \\
\hline \multirow[t]{3}{*}{ Substâncias nocivas não especificadas (X69) } & 2.935 & 6,5 & 3.136 & 9,1 & 6.071 & 7,6 \\
\hline & \multicolumn{2}{|c|}{$\begin{array}{l}\text { Homem } \\
(\mathrm{n}=1.560)\end{array}$} & \multicolumn{2}{|c|}{$\begin{array}{l}\text { Mulher } \\
(\mathrm{n}=842)\end{array}$} & \multicolumn{2}{|c|}{$\begin{array}{c}\text { Total } \\
(\mathrm{n}=2.402)\end{array}$} \\
\hline & $\mathbf{n}$ & $\%$ & $\mathbf{n}$ & $\%$ & $\mathbf{n}$ & $\%$ \\
\hline \multicolumn{7}{|l|}{ Óbitos } \\
\hline Medicamentos (X60-X64) & 408 & 26,2 & 388 & 46,1 & 796 & 33,1 \\
\hline Álcool (X65) & 454 & 29,1 & 66 & 7,8 & 520 & 21,6 \\
\hline $\begin{array}{l}\text { Outros sólidos, líquidos, gases e vapores } \\
\text { (X66-X67) }\end{array}$ & 36 & 2,3 & 12 & 1,4 & 48 & 2,0 \\
\hline Pesticidas (X68) & 478 & 30,6 & 255 & 30,3 & 733 & 30,5 \\
\hline Substâncias nocivas não especificadas (X69) & 184 & 11,8 & 121 & 14,4 & 305 & 12,7 \\
\hline
\end{tabular}

Fonte: SIH-SUS/DATASUS/MS

*As variáveis faixa etária (15 a 19 anos) e categorias de causas (medicamentos) apresentaram apenas um único registro como ignorado cada, 0,01\% e $0,003 \%$, respectivamente
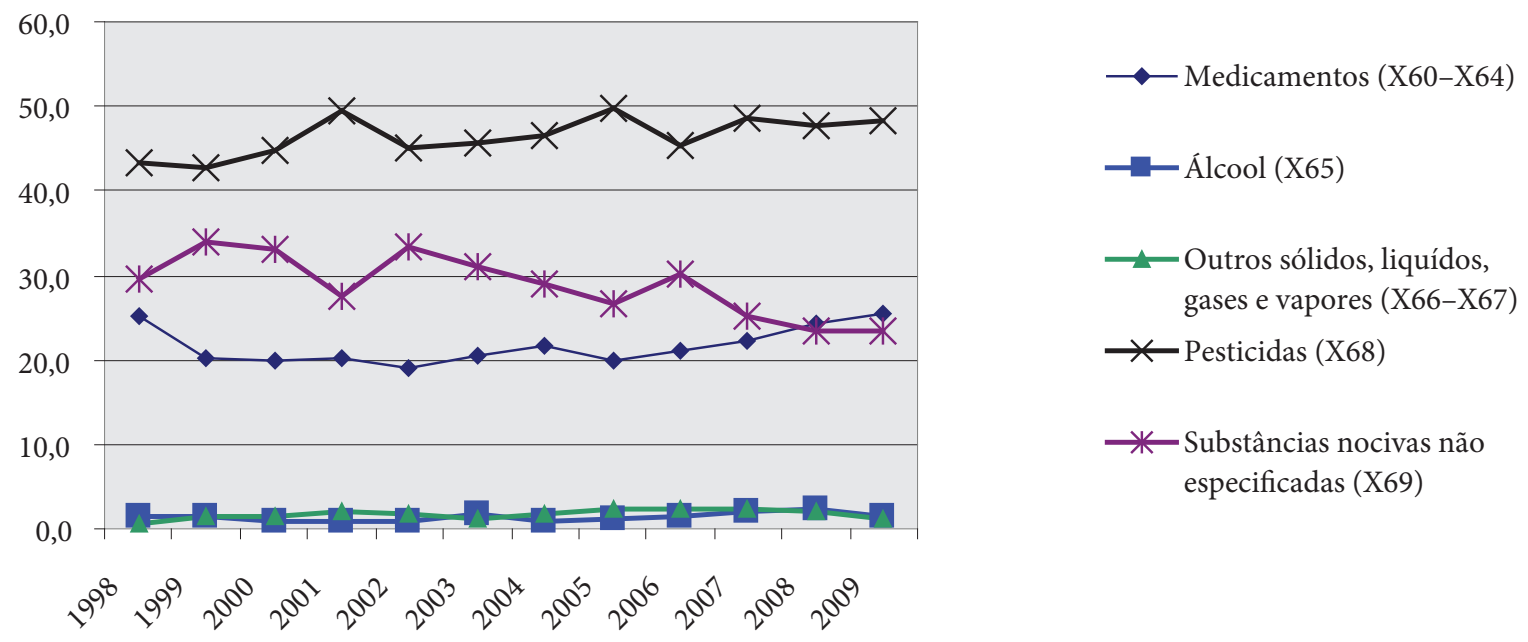

Fonte: SIM/DATASUS/MS

Gráfico 2. Distribuição da frequência de suicídios devido à intoxicação exógena, segundo categorias de substâncias, no Brasil, período de 1998 a 2009 
Tabela 2. Distribuição da frequência de suicídio devido à intoxicação exógena, segundo sexo, Brasil, período de 1998 a 2009

\begin{tabular}{|c|c|c|c|c|c|c|}
\hline & \multicolumn{2}{|c|}{$\begin{array}{c}\text { Homem } \\
(\mathrm{n}=8.953)\end{array}$} & \multicolumn{2}{|c|}{$\begin{array}{c}\text { Mulher } \\
(\mathrm{n}=5.504)\end{array}$} & \multicolumn{2}{|c|}{$\begin{array}{c}\text { Total } \\
(\mathrm{n}=14.457)\end{array}$} \\
\hline & $\mathbf{n}$ & $\%$ & $\mathrm{n}$ & $\%$ & $\mathbf{n}$ & $\%$ \\
\hline \multicolumn{7}{|l|}{ Faixa Etária (anos) } \\
\hline 10 a 14 & 47 & 0,5 & 176 & 3,2 & 223 & 1,5 \\
\hline 15 a 19 & 463 & 5,2 & 837 & 15,2 & 1.300 & 9,0 \\
\hline 20 a 29 & 2.071 & 23,1 & 1.323 & 24,0 & 3.394 & 23,5 \\
\hline 30 a 39 & 2.179 & 24,3 & 1.224 & 22,2 & 3.403 & 23,5 \\
\hline 40 a 49 & 1.936 & 21,6 & 1.057 & 19,2 & 2.993 & 20,7 \\
\hline 50 a 59 & 1.154 & 12,9 & 520 & 9,4 & 1.674 & 11,6 \\
\hline 60 e mais & 1.103 & 12,3 & 367 & 6,7 & 1.470 & 10,2 \\
\hline \multicolumn{7}{|l|}{ Categoria de causas } \\
\hline Medicamentos (X60-X64) & 1.552 & 17,3 & 1.589 & 28,9 & 3.141 & 21,7 \\
\hline Álcool (X65) & 153 & 1,7 & 54 & 1,0 & 207 & 1,4 \\
\hline Outros sólidos, líquidos, gases e vapores (X66-X67) & 191 & 2,1 & 68 & 1,2 & 259 & 1,8 \\
\hline Pesticidas (X68) & 4.400 & 49,2 & 2.351 & 42,7 & 6.751 & 46,7 \\
\hline Substâncias nocivas não especificadas (X69) & 2.657 & 29,7 & 1.442 & 26,2 & 4.099 & 28,4 \\
\hline
\end{tabular}

Fonte: SIM/DATASUS/MS

\section{DISCUSSÃO}

Como limitação do estudo, os diferentes tipos clínicos de intoxicação (aguda, subaguda e crônica) não são considerados nos sistemas, portanto, não entraram nas análises. Este fato, entretanto, não altera o perfil dos casos analisados, já que pode ser compreendido através da informação sobre a evolução do caso, isto é ir a óbito ou não. Outro aspecto é o fato de os registros do SIH-SUS serem apenas de tentativas de suicídio que precisaram de internação, perdendo-se informação sobre os demais casos. A subnotificação se deve a questões culturais/ religiosos e legais, como mencionado anteriormente. $\mathrm{O}$ erro de classificação é outro aspecto importante para a subnotificação causada, por exemplo, por imprecisões encontradas na determinação dos casos em estudos de mortalidade ${ }^{23}$. Estudo realizado na Suécia mostrou que a incerteza na classificação sobre o tipo de morte estaria ligada a causas como envenenamento e afogamento, devido à variação nas impressões do legista. As orientações para apoiar a investigação policial e o exame médico-legal devem ser subsidiadas por uma coleta de dados primários, com boa qualidade e em todas as fases da investigação de uma morte não natural, visando assim a redução do número de casos indeterminados para possíveis suicídios $^{24}$. O presente estudo buscou contornar suas limitações quanto às subnotificações e erros de classificação, havendo trabalhado com diferentes fontes oficiais.

Nossos resultados mostraram que a intoxicação exógena é a primeira causa de internação por tentativas de suicídio e a segunda causa de óbito por suicídio, segundo os sistemas de informação analisados. No caso das tentativas de suicídio, o número total de homens que tentaram suicídio foi maior do que o de mulheres a partir dos 20 anos de idade, no período considerado, contrariando a literatura que sempre apontou uma sobremorbidade feminina ${ }^{3,6,25-27}$. Quanto aos dados do SIM, permanece a sobremortalidade masculina, principalmente a partir dos 20 anos de idade. Já segundo o óbito hospitalar (SIH-SUS), a sobremortalidade é feminina. Esta mortalidade não é, em geral, a considerada pelos autores que utilizam o SIM como a fonte de referência para a mortalidade por suicídio. Outra questão que surpreende é que, embora o número de tentativas de suicídio entre as adolescentes seja reconhecidamente mais elevado do que naqueles do sexo oposto, o número de óbitos entre elas também foi mais elevado, o que não é o esperado. Esses resultados levam a pensar em possíveis mudanças no padrão de comportamento suicida deste grupo.

A letalidade (probabilidade de morte) é um aspecto importante nos meios utilizados para suicídio e tentativas. Estudos mostram uma sobremorbidade feminina nas tentativas de suicídio, enquanto os homens cometeriam mais suicídio devido ao maior uso de métodos letais ${ }^{26,28}$. Pesquisas recentes têm mostrado que a sobremortalidade masculina tem estado presente mesmo em métodos considerados menos letais ou que permitam maior chance de socorro, tais como a intoxicação exógena ${ }^{14,29,30}$. Existem hipóteses que sugerem que a diferença na escolha de métodos mais letais pelos homens seria a maior intenção de morrer, impulsividade, agressividade, uso de álcool, isolamento social, demora em buscar ajuda, além de fatores socioeconômicos, como o desemprego que poderia favorecer à depressão e ansiedade ${ }^{3,17,31}$. Estas constatações apontam a necessidade de se investigar outros fatores 
que seriam intrínsecos à escolha do método para o suicídio, tendo em conta que possivelmente seja o grau da letalidade que diferencie os dois agravos.

Inúmeros estudos já chamaram a atenção para a gravidade do uso indiscriminado tanto de medicamentos quanto de pesticidas ${ }^{2-4,25,32}$. Entretanto, só conseguimos contribuir para esta questão, neste estudo, apontando que os óbitos femininos estão se igualando aos masculinos quando se trata dos suicídios por pesticidas. O presente estudo não responde a esta possível mudança no padrão das intoxicações, mas levanta questões para trabalhos futuros.

A aceitabilidade social também tem sido apontada como importante papel na escolha dos métodos utilizados nos suicídios e tentativas. Alguns autores argumentam que a letalidade do método não estaria diretamente relacionada à intenção de morte em si, mas a uma aceitação social que teria o gênero como base para a escolha do método suicida. Deste modo, a escolha das mulheres pelo uso de medicamentos como meio de suicídio seria socialmente mais aceito do que para os homens, dos quais "se esperaria" um meio mais violento como enforcamento ou uso de arma de fogo ${ }^{17,27}$. A combinação em certo grau entre aceitabilidade social e disponibilidade ao método para o suicídio são importantes aspectos para a escolha, sendo pouco provável que um método seja escolhido sem um desses dois aspectos ${ }^{13,33}$. O predomínio apontado por diversos estudos sobre o uso de medicamentos pelas mulheres parece corroborar esta argumentação, uma vez que mesmo sendo acessíveis a ambos os sexos, as mulheres utilizam mais os medicamentos.

A disponibilidade do método escolhido pelo indivíduo tem sido apontada em diversos estudos como outro importante fator de risco para o suicídio e tentativas ${ }^{13,17,33,34}$. A questão com o tema disponibilidade é o fato de que não se trata de controlar todos os meios utilizados, inclusive porque isto seria impossível, mas sim, reduzir ao máximo o fácil acesso. No caso da intoxicação exógena como método, alguns estudos apontam resultados importantes quando diminuíram a toxicidade de alguns produtos como gases e pesticidas, além de medidas de proteção em frascos de medicamentos, por exemplo.

O uso de pesticidas nos suicídios e tentativas é marcante nos países asiáticos e da América Latina, representando uma frequência entre 60 a $90 \%{ }^{35}$, principalmente em áreas rurais. E se este fato é comum nos países em desenvolvimento, nos países industrializados as substâncias mais utilizadas na intoxicação são os medicamentos. O conhecimento dos métodos é importante para se pensar na prevenção. AjdacicGross et al..$^{33}$ analisaram dados da Organização Mundial de Saúde (OMS) e identificaram uma polarização entre o suicídio por pesticidas e arma de fogo, enquanto que o enforcamento teria uma posição intermediária. Uma hipótese para esta polarização do uso de pesticidas e arma de fogo seria o impacto da disponibilidade em relação aos outros métodos, além de facilitarem os suicídios não planejados, baseados no impulso, por exigirem pouco conhecimento técnico, rápido uso e alta letalidade.

Já Gunnell e Eddleston ${ }^{8}$ trazem a discussão sobre a maior frequência de suicídio por uso de pesticidas em países em desenvolvimento devido ao modelo de prática agrícola, a qual favoreceria um maior número de pessoas manipulando os produtos, além de os armazenarem próximo às residências, inclusive, dentro das mesmas. Isto facilitaria tanto a disponibilidade quanto aceitabilidade no uso das substâncias nos suicídios. Os autores também argumentam que, tendo os impulsos suicidas geralmente uma curta duração, dificultar o acesso ou reduzir os aspectos mais letais dos meios que são utilizados podem reduzir uma importante proporção desses agravos. Trabalho desenvolvido por Gunnell et al. ${ }^{34}$ sugere, utilizando o exemplo de Sri Lanka, que foi através da restrição de agrotóxicos classe I (extremamente tóxico) que ocorreu o declínio das taxas de suicídio. Os autores não associam diretamente estes declínios nas taxas de condições sociais e econômicas, tais como a taxa de desemprego, o abuso de álcool, divórcio, o próprio uso de pesticidas e os anos de guerra civil. Assim seria o alto grau de toxicidade do pesticida que faria a diferença, isto é seria o grande "vilão".

Em 2008, o Brasil tornou-se o maior consumidor de agrotóxico do mundo e em 2010, o mercado nacional representou $19 \%$ do mercado mundial de agrotóxicos, seguido pelos Estados Unidos com 17\%. Em recente comunicado da Agência Nacional de Vigilância Sanitária (ANVISA), apenas cerca de 50\% dos agrotóxicos registrados no Brasil estão disponíveis aos agricultores ${ }^{36}$.

A relação entre tentativa/suicídio e medicamentos vem sendo mostrada mais frequentemente em estudos realizados em países desenvolvidos ${ }^{6,8,37}$, onde se observa o pouco uso dos pesticidas com esta finalidade. Estudos brasileiros falam sobre a importância tanto dos medicamentos quanto dos pesticidas nesta relação, ocorrendo, entretanto, em nosso meio, sobremortalidade feminina no suicídio por uso de medicamentos ${ }^{2-4,38}$. O estudo de Nordentoft ${ }^{39}$ aponta a gravidade da pouca importância que vem sendo dada à restrição de medicamentos relacionados à alta letalidade, tais como barbitúricos, antidepressivos tricíclicos e outros. A autora chama a atenção para a necessidade da inclusão do tema nas estratégias de prevenção do suicídio.

Continuando esta discussão, outros estudos nacionais trazem informações sobre o hábito dos brasileiros em manterem 
estoques domiciliares de medicamentos, o que favorece o acesso para suicídios e tentativas ${ }^{40,41}$. O Projeto de Lei no 7.029 de $2006^{42}$ visa garantir a obrigatoriedade na venda de medicamentos fracionados, sendo medida de provável impacto no comportamento do brasileiro de armazenar medicamentos. Contudo, em nosso meio este cenário parece longínquo, haja vista que tal projeto ainda tramita na Câmara Federal ${ }^{2}$. No Brasil, a ANVISA já regulamentou o medicamento fracionado, porém sem obrigatoriedade ( $\mathrm{RDC} \mathrm{n}^{\circ} 80$, de 11 de maio de 2006) ${ }^{43}$. Assim, ainda avançamos pouco nas medidas de prevenção e restrição.

Outro ponto importante é o do descarte de medicamentos vencidos ou não usados pela população. Embora aprovada em agosto de 2010, a Lei no 12.305 , que institui a Política Nacional de Resíduos Sólidos (PNRS), avançou pouco em medidas adequadas para o descarte dos medicamentos. Em 2011, foi instituído o Grupo de Trabalho Temático (GTT), composto pela Federação Nacional dos Farmacêuticos (FENAFAR), outros representantes do movimento social, setor empresarial e do Poder Público, sob a coordenação do Ministério da Saúde e da ANVISA. Este GTT visa a construção de um modelo que permita a correta devolução dos medicamentos pelo usuário até o seu descarte ambiental correto. Soma-se aos esforços a aprovação do Projeto de Lei no 595 de 2011, o qual propõe que as farmácias, drogarias e postos de saúde seriam obrigados a receber os medicamentos devolvidos pela população (vencidos ou não) e estes produtos seriam remetidos ao laboratório que os produziu, realizando então o descarte ${ }^{44,45}$.

Outra substância que chamou atenção foi o álcool que, frequentemente, é relacionado a abuso/dependência. Ao trabalharmos com os dados de internação por suicídio e tentativas (SIH-SUS), vimos a sua importância não só sobre a morbidade masculina, como também sobre a frequência dos óbitos hospitalares, o que não pode ser observado ao se considerar unicamente o SIM. Estudos internacionais mostram que de 20 a $40 \%$ dos suicídios e tentativas do sexo masculino ocorreram em indivíduos sob o efeito do álcool no momento da tentativa ${ }^{46-48}$. Esses resultados não foram diferentes daqueles encontrados nos estudos nacionais, cujas taxas ficaram entre 26 e $37 \% \%^{3,25,49}$. Fudalej et al. ${ }^{46}$ constataram que indivíduos sob a influência do álcool, e utilizando diferentes meios para o suicídio, apresentam chance 4,6 vezes maior de se suicidar do que aqueles que não beberam, isto é, o suicídio sob a influência do álcool está fortemente relacionado com dependência.

Outro olhar sobre a relação entre álcool e suicídio mostra que o consumo excessivo de álcool e a intoxicação alcoólica aguda levam a uma perda de inibição, comportamento impulsivo, além de uma tendência a assumir riscos. Este quadro aumenta em até seis vezes o risco de suicídio ${ }^{48,50}$.
O uso abusivo de álcool pela população masculina brasileira $^{51,52}$, fez com que, pela primeira vez, o governo brasileiro criasse estratégias ampliadas a várias drogas, dentre elas o álcool, previsto no Plano Integrado de Enfrentamento ao Crack e Outras Drogas (PIEC) (Decretolei $\left.\mathrm{n}^{\circ} 7.179 / 2010\right)^{53,54}$. Estas ações são importantes para o enfrentamento dos problemas causados pelo uso de álcool e drogas que impactam fortemente a sociedade brasileira e, em especial, o setor saúde por sua associação com acidentes de trânsito, homicídios e suicídios².

\section{CONCLUSÃO}

Este artigo se propôs descrever frequências relativas dos casos suicídios/tentativas por intoxicação exógena registrados nos sistemas oficiais, utilizando essas informações como referência para uma discussão sobre acesso e medidas restritivas às substâncias usadas nos agravos. Diferentemente dos achados de outros estudos, ressalta-se aqui que os homens tentaram mais o suicídio do que as mulheres e quanto aos óbitos hospitalares, os homens também se destacaram quando o meio utilizado foi o álcool. Também chama a atenção que, quando estes óbitos foram determinados pelos pesticidas, as frequências para ambos os sexos se igualaram.

$\mathrm{Na}$ leitura dos planos estratégicos, observou-se que estes não são planos direcionados aos agravos aqui analisados, mas indicativos de algumas ações sobre fatores que favorecem os comportamentos suicidas, como por exemplo, a assistência para os casos de usuários de substâncias psicoativas. Os planos não indicam claramente ações intersetoriais e sinérgicas, são pontuais e não configuram um plano de fato para a prevenção do suicídio. Na prática, não há nenhuma restrição efetiva aos meios, seja por falta de iniciativa ou por morosidade no processo legal, como o caso do fracionamento dos medicamentos. A literatura mostra que vários países, também em desenvolvimento como o nosso, iniciaram o processo de restrição ao uso destas substâncias quando altamente tóxicas e já exibiram resultados, como Índia e Sri-Lanka. Por último não podemos deixar de citar o caso do álcool, droga lícita, de aceitabilidade social ampla, especialmente entre os jovens, cuja proposta para sua prevenção não considera os suicídios. Nestes, o álcool pode ser um meio, um favorecedor do impulso suicida, fator preditor quando ocorre a dependência dessa substância e, ainda, comorbidade de outros transtornos mentais, aumentando expressivamente o risco de suicídio. Constata-se a necessidade de se considerar o comportamento suicida como de fato um problema de saúde pública em ascensão no Brasil e investir em políticas públicas para sua prevenção. 


\section{REFERÊNCIAS}

1. Spiller HA, Appana S, Brock GN. Epidemiological trends of suicide and attempted suicide by poisoning in the US: 2000-2008. Leg Med (Tokyo). 2010;12(4):177-83.

2. Santos SA, Legay, LF, Lovisi GM, Santos JFC, Lima LA. Tentativas e suicídios por intoxicação exógena no Rio de Janeiro: análise dos dados dos sistemas oficiais de informação em saúde, 2006-2008. Rev Bras Epidemiol. No prelo 2012.

3. Santos SA, Lovisi G, Legay L, Abelh L. Prevalência de transtornos mentais nas tentativas de suicídio em um hospital de emergência no Rio de Janeiro, Brasil. Cad Saúde Pública. 2009;25(9):2064-74.

4. Lovisi GM, Santos SA, Legay L, Abelha L, Valencia E. Epidemiological analysis of suicide in Brazil from 1980 to 2006. Rev Bras Psiquiatr. 2009; 31(Suppl. I2): S86-93.

5. Damas FB, Zannin M, Serrano AI. Tentativas de suicídio com agentes tóxicos: análise estatística dos dados do CIT/SC (1994 a 2006). Rev Bras Toxicol. 2009;22(1-2):21-6.

6. Caballero Vallés PJ, Dorado Pombo S, Díaz Brasero A, García Gil ME, Yubero Salgado L, Torres Pacho N, et al. Vigilancia epidemiológica de la intoxicación aguda en el área sur de la Comunidad de Madrid: estudio VEIA 2004. An Med Interna (Madrid). 2008;25(6):262-8.

7. Bossuyt N, Van Casteren V. Epidemiology of suicide and suicide attempts in Belgium: results from the sentinel network of general practitioners. Int J Public Health. 2007;52(3):153-7.

8. Gunnell D, Eddleston M. Suicide by intentional ingestion of pesticides: a continuing tragedy in developing countries. Int J Epidemiol. 2003;32(6):902-9.

9. World Health Organization; Krug EG et al. (ed.). World report on violence and Health. Geneva: WHO; 2002.

10. Marín-León L, Barros MB. Mortes por suicídio: diferenças de gênero e nível socioeconômico. Rev Saude Publica. 2003;37(3):357-63.

11. Minayo MC. Suicídio: Violência auto-infligida. In: Ministério da Saúde, Secretaria de Vigilância em Saúde. Impactos da violência na saúde dos brasileiros. Brasília: Ministério da Saúde; 2005.

12. Pritchard C, Heal S. Suicide and undetermined deaths among youths and young adults in Latin America: comparison with the 10 major developed countries - a source of hidden suicides? Crisis. 2008;29(3):145-53.

13. Cantor $\mathrm{CH}$, Baume PJM. Access to methods of suicide: what impact? Aust N Z J Psychiatry. 1998;32(1):8-14.

14. Cibis A, Mergl R, Bramesfeld A, Althaus D, Niklewski G, Schmidtke A, et al. Preference of lethal methods is not the only cause for higher suicide rates in males. J Affect Disord. 2012;136 (1-2):9-16.

15. Bergen H, Hawton K, Waters K, Ness Jennifer, Cooper J, Steeg S, et al. How do methods of non-fatal self-harm relate to eventual suicide? J Affect Disord. 2012;136(3):526-33.

16. Berecz R, Cáceres M, Szlivka A, Dorado P, Bartók E, Peñas-LLedó E, et al. Reduce completed suicide rate in Hungary from 1990 to 2001: relation to suicide methods. J Affect Disord. 2005;88(2):235-8.

17. Hawton K. Restricting access to methods of suicide rationale and evaluation of this approach to suicide prevention. Crisis. 2007;28:4-9.

18. Buckley NA, Gunnell D. Does restricting pack size of paracetamol (acetaminophen) reduce suicides? PLoS Med. 2007;4(4):e152.
19. Larini L.Toxicologia. São Paulo: Manole; 1987.

20. Organização Mundial de Saúde (OMS). Classificação Internacional de Doenças e Problemas Relacionados à Saúde. Centro Colaborador da OMS para a Classificação de Doenças em Português, tradução. 10 ed. rev. São Paulo: Edusp; 2007.

21. Carvalho DMT. Sistema de Informações Hospitalares do SUS - SIHSUS. In: Ministério da Saúde (BR), Organização Pan-Americana da Saúde, Fundação Oswaldo Cruz. A experiência brasileira em sistemas de informação em saúde. Brasília: Ministério da Saúde; 2009.

22. Mello Jorge MHP, Laurenti R, Gotlieb SLDO. Sistema de informações sobre Mortalidade - SIM: Concepção, implantação e avaliação. In: Ministério da Saúde (BR), Organização Pan-Americana da Saúde, Fundação Oswaldo Cruz. A experiência brasileira em sistemas de informação em saúde. Brasília: Ministério da Saúde; 2009.

23. Sainsbury P, Jenkins JS. The accuracy of officially reported suicide statistics for purposes of epidemiological research. J Epidemiol Community Health. 1982;36(1):43-8.

24. Lindqvist P, Gustafsson L. Suicide classification - clues and their use. A study of 122 cases of suicide and undetermined manner of death. Forensic Sci Int. 2002;128(3):136-40.

25. Stefanello S, Cais CFS, Mauro MLF, Freitas GVS, Botega NJ. Gender differences in suicide attempts: preliminary results of the multisite intervention study on suicidal behavior (SUPRE-MISS) from Campinas, Brazil. Rev Bras Psiquiatr. 2008;30(2):139-43.

26. Beautrais AL. Women and suicidal behavior. Crisis. 2006;27(4):153-6.

27. Canetto SS, Sakinofsky I. The gender paradox in suicide. Suicide Life Threat Behav. 1998;28(1):1-23.

28. Hawton K. Sex and suicide: gender differences in suicidal behaviour. $\mathrm{Br} \mathrm{J}$ Psychiatry. 2000;177:484-5.

29. Elnour AA, Harrison J. Lethality of suicide methods. Inj Prev. 2008;14(1):39-45.

30. Nishimura A, Shioiri T, Nushida H, Ueno Y, Ushiyama I, Tanegashima A, et al. Changes in choice of method and lethality between last attempted and completed suicides: How did suicide attempters carry out their desire? Leg Med (Tokyo). 1999;1(3):150-8.

31. Schneider B, Grebner K, Schnabel A, Hampel H, Georgi K, Seidler A. Impact of employment status and work-related factors on risk of completed suicide. A case-control psychological autopsy study. Psychiatry Res. 2011;190 (2-3):265-70.

32. Werneck GL, Hasselmann MH, Phebo LB, Vieira DE, Gomes VLO (2006). Tentativas de suicídio em um hospital geral no Rio de Janeiro, Brasil. Cad Saúde Pública. 2006;22(10):2201-6.

33. Ajdacic-Gross V, Weiss MG, Ring M, Hepp U, Bopp M, Gutzwiller F; et al. Methods of suicide: international suicide patterns derived from the WHO mortality database. Bull World Health Organ. 2008; 86(9):726-732.

34. Gunnell D, Fernando R, Hewagama M, Priyangika WDD, Konradsen F, Eddleston M. The impact of pesticide regulations on suicide in Sri Lanka. Int J Epidemiol. 2007;36:1235-42.

35. Bertolote JM, Fleischmann A, Butchart A, Besbelli N. Suicide, suicide attempts and pesticides: a major hidden public health problem. Bull World Health Organ. 2006; 84(4):260. 
36. Molina, D. Agricultor não tem acesso a 50\% dos agrotóxicos registrados. [cited 2012 April 11]. Available from: http://portal.anvisa. gov.br/wps/content/anvisa+portal/anvisa/sala+de+imprensa/menu++ noticias+anos/2012+noticias/seminario+volta+a+discutir+mercado+ de+agrotoxicos $+\mathrm{em}+2012$

37. Värnik A, Kõlves K, Allik J, Arensman E, Aromaa E, van Audenhove $\mathrm{C}$, et al. Gender issues in suicide rates, trends and methods among youths aged 15-24 in 15 European countries. J Affect Disord. 2009;113(3):216-226.

38. Bochner R. Perfil das intoxicações em adolescentes no Brasil no período de 1999 a 2001. Cad Saúde Pública. 2006;22(3):587-95.

39. Nordentoft M. Restrictions in availability of drugs used for suicide. Crisis. 2007;28(Suppl 1):44-9.

40. Fernandes LC. Caracterização e análise da farmácia caseira ou estoque domiciliar de medicamentos. [d issertação]. Porto Alegre (RS): Universidade Federal do Rio Grande do Sul; 2000.

41. Ribeiro MA, Heineck I. Estoque Domiciliar de Medicamentos na Comunidade Ibiaense Acompanhada pelo Programa Saúde da Família, em Ibiá-MG, Brasil. Saúde Soc. São Paulo. 2010;19(3):653-63.

42. Brasil. Projeto de Lei ${ }^{\circ} 7.029 / 2006$. Acresce dispositivos ao art. $22 \mathrm{da}$ Lei $\mathrm{n}^{\circ} 6.360$, de 23 de setembro de 1976, para dispor sobre registro e fracionamento de medicamentos para dispensação, e dá outras providências. [cited 2012 April 5]. Available from: http://www.camara. gov.br/proposicoesWeb/fichadetramitacao?idProposicao $=324349$.

43. Brasil. Resolução da Diretoria Colegiada ${ }^{\circ}$ 80, de 11 de maio de 2006. [cited 2012 April 5]. Available from: http://www.anvisa.gov.br/hotsite/ fraciona/rdc_80.htm

44. Federação Nacional dos Farmacêuticos (FENAFAR). Medicamento vencido deve ser descartado em postos de coleta. [cited 2012 Dec 15]. Available from: http://www.fenafar.org.br/portal/medicamentos/62medicaments/1193-medicamento-vencido-deve-ser-descartado-empostos-de-coleta.html

45. Federação Nacional dos Farmacêuticos (FENAFAR). [cited 2012 Dec 15]. Available from: http://www.fenafar.org.br/portal/images/ stories/pdf/docdebate.pdf
46. Fudalej S, Ilgen M, Fudalej M, Wojnar M, Matsumoto H, Barry K L, et al. Clinical and genetic risk factors for suicide under the influence of alcohol in a Polish sample. Alcohol Alcohol. 2009;44(5):437-42.

47. Boenisch S, Bramesfeld A, Mergl R, Havers I, Althaus D, Lehfeld H, et al. The role of alcohol use disorder and alcohol consumption in suicide attempts - A secondary analysis of 1921 suicide attempts. Eur Psychiatry. 2010;25(7):414-20.

48. Holmgren A, Jones AW. Demographics of suicide victims in Sweden in relation to their blood-alcohol concentration and the circumstances and manner of death. Forensic Sci Int. 2010; 198(1-3):17-22.

49. Ponce JC, Andreuccetti G, Jesus MGS, Leyton V, Muñoz DR. Álcool em vítimas de suicídio em São Paulo. Rev Psiquiatr Clín. 2008;35 (Suppl 1):13-6.

50. Harris EC, Barraclough B. Suicide as an outcome for mental disorders: a meta-analysis. Br J Psychiatry. 1997;170(3):205-28.

51. Carlini EA, Galduróz JC, Noto AR, Carlini CM, Oliveira LG, Nappo SA, et al. II Levantamento domiciliar sobre o uso de drogas psicotrópicas no Brasil: estudo envolvendo as 108 maiores cidades do país: 2005. São Paulo: CEBRID: UNIFESP, 2006

52. Guimarães VV, Florindo AA, Stopa SR, Galvão César CL, Barros MBA, Carandina $\mathrm{L}$, et al. Consumo abusivo e dependência de álcool em população adulta no Estado de São Paulo, Brasil. Rev Bras Epidemiol. 2010;13(2):314-25.

53. Brasil. Presidência da República. Secretaria de Imprensa. Plano Integrado de Enfrentamento ao Crack e Outras Drogas investe R \$ 400 milhões em ações de saúde, assistência e repressão ao tráfico. [cited 2011 Feb 8]. Available from: http://cbdd.org.br/pt/files/2009/10/PIEC.pdf.

54. Brasil. Decreto $\mathrm{n}^{\circ} 7.179$, de 20 de maio de 2010. Institui o Plano Integrado de Enfrentamento ao Crack e outras Drogas, cria o Comitê Gestor e dá outras providências. Diário Oficial da União no 96 , seção 1 , p. 34, de 21 de maio de 2010. [cited 2013 April 4]. Available from: http:// www.in.gov.br/imprensa/visualiza/index.jsp?.jornal $=1$ \&pagina $=43 \&$ da ta $=21 / 05 / 2010$

Recebido em: 22/07/2012 Aprovado em: 08/01/2013 